\title{
Damage tolerance analysis of aircraft reinforced panels
}

\author{
F. Carta, A. Pirondi \\ Industrial Engineering Department, University of Parma, v.le G.P. Usberti 181/ A, 43124 Parma - Italy
}

\begin{abstract}
This work is aimed at reproducing numerically a campaign of experimental tests performed for the development of reinforced panels, typically found in aircraft fuselage. The bonded reinforcements can significantly reduce the rate of fatigue crack growth and increase the residual strength of the skin. The reinforcements are of two types: stringers and doublers. The former provides stiffening to the panel while the latter controls the crack growth between the stringers. The purpose of the study is to validate a numerical method of analysis that can predict the damage tolerance of these reinforced panels. Therefore, using a fracture mechanics approach, several models (different by the geometry and the types of reinforcement constraints) were simulated with the finite element solver ABAQUS. The bonding between skin and stiffener was taken either rigid or flexible due to the presence of adhesive. The possible rupture of the reinforcements was also considered. The stress intensity factor trend obtained numerically as a function of crack growth was used to determine the fatigue crack growth rate, obtaining a good approximation of the experimental crack propagation rate in the skin. Therefore, different solutions for improving the damage tolerance of aircraft reinforced panels can be virtually tested in this way before performing experiments.
\end{abstract}

KEYWORDS. Aircraft stiffened panels; Fatigue crack growth; Damage tolerance analysis.

\section{INTRODUCTION}

$\mathrm{I}$ n aircraft fuselage, aluminum stiffeners are connected to panel in longitudinal and circumferential directions. A particularly significant application is the direct bonding between stringers and the surface of the fuselage skin. The main features of the reinforced bonded panels concern better damage tolerance and higher stability at different types of loads $[1,2]$.

The experiments on aluminum panels with bonded stiffeners show that a limit of the aluminum reinforcement is the premature rupture of the reinforcement caused by the load transfer from the skin to the stiffeners when the crack runs underneath it. To improve the tolerance to the fracture, the doublers or reinforcement cords should preferably be made of material resistant to fatigue, with high stiffness and static strength $[3,4]$.

Panels made of a thin metal skins stiffened with bonded reinforcements insensitive to fatigue, can ensure slow crack propagation if not its arrest, and the capability to withstand a large damage, combined with a low structural weight. The effects of this bonded reinforcements or doublers are very difficult to predict numerically or analytically, because of the complex mechanisms of failure:

- separation at the interface between skin and reinforcement around the area of nucleation and propagation of the crack;

- load redistribution between the damaged and undamaged reinforcement;

- fatigue damage of the reinforcement which may cause his premature rupture;

- crack bridging by the doublers thanks if they have a sufficiently high fatigue strength. 
In addition, secondary effects, such as residual stresses generated by the bonding process and bending caused by the eccentricity of the load with respect to the neutral axis of the reinforced panel cross-section, increase the complexity of the phenomenon. Reliable predictions of crack growth and residual strength in bonded structures can be based mainly on empirical considerations. The experimental results which support the numerical analysis reported in this work refer to an experimental investigation carried out by Airbus in a period from 2002 to 2007. Through an extensive campaign of tests, several methods of reinforcement were analyzed, using bonded reinforcements in the fuselage panels. To achieve a quantitative study, in the analysis different types of connection between the reinforcements and the skin were considered. In the literature, numerical studies on FCP (Fatigue Crack Propagation) in reinforced structures are available. However, if the damage tolerance assessments appear to be practicable in integral reinforced structures [5], the same assessment is not straightforward in differential structures with bonded joints between skin and chords [6, 7] due to the complex mechanisms mentioned previously.

\section{STIFFENED DIFFERENTIAL STRUCTURES}

he stiffened differential structures can actually be reproduced with proper models that allow replicating the stiffener effect using the "crack arrest" philosophy design. In this approach after an initial propagation, the crack arrests due to a stiffener when a given length is reached (see Fig. 1).

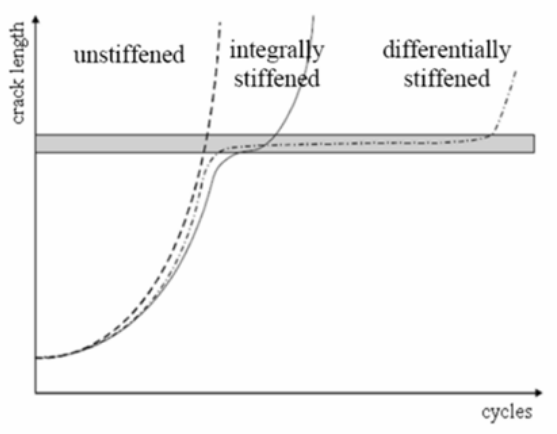

Figure 1: Comparison between the several design solutions according the "crack arrest" method.

In the differential structures the crack propagates typically only in the skin, then the completely intact stiffeners can control the defect evolution during the propagation, due to the load transfer from the skin to the stiffeners which, in turn, means that the stress intensity factor decreases. This fact underlines the effectiveness design of crack arrest. Several experiments have shown a significant beneficial effect due to the presence of reinforcements in differential structures [1, 2].

In many engineering fields such as aeronautics, automotive, marine or civil engineering, plates and shell made with laminate composite structures are largely used in load-bearing structural members. Important examples of these applications can be observed in aerospace engineering, where thin laminates are reinforced by a certain number of profiles (the so called stringers).

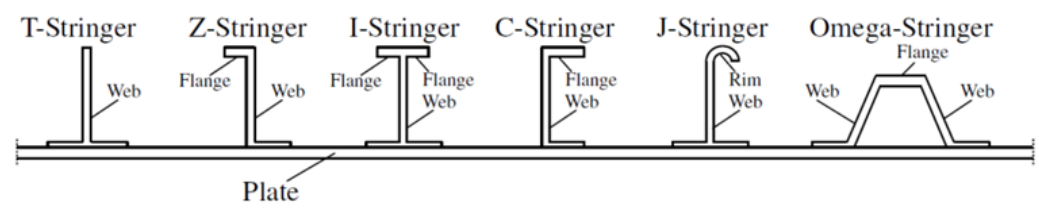

Figure 2: Cross sections of several kinds of stringers.

Such structural parts can readily be found in fuselages, tail planes, or wind covers of aircrafts and typically consist of a thin plate or moderately curved thin shell that is stiffened by a certain number of shaped stringers (see Fig. 2). Especially stringers which have a closed-profile cross-section may provide a high torsional stiffness to the plate such that the composite plate itself can be assumed to be elastically restrained to some degrees [8]. 
Another kind of reinforcement, called doubler, is usually positioned in the separation zone between stringers, oriented parallel to them (see Fig. 3).

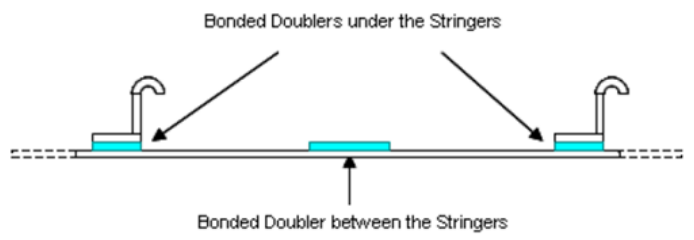

Figure 3: Representations of the possible placements of the doublers: in the middle of the bay (between the stringers) or under the stringers.

Because of their shape, the doublers have not a stiffening function, but they slow down the crack grow rate in the zone between the stringers (the so-called bays). The doublers are typically bonded to the skin and experimental studies are showed they are more efficient with a thick section rather than a thin section [2].

\section{EXPERIMENTS}

A total of 35 stiffened panels, representative of a typical fuselage skin of a long-range family aircraft, were manufactured and tested in the laboratories of EADS-IW Ottobrunn. The fatigue crack propagation (FCP) rate was investigated for twenty-four of them. The remaining eleven panels were tested for the residual strength.

The panel shown in Fig. 4 has been tested and it is characterized by a skin (1224 mm wide and $1455 \mathrm{~mm}$ long) with seven equally spaced bonded stringers. In addition to the stringers, bonded doublers are positioned below and between the stringers in order to provide additional reinforcement. All the doublers are placed orthogonal to the direction of crack propagation alike the stringers.

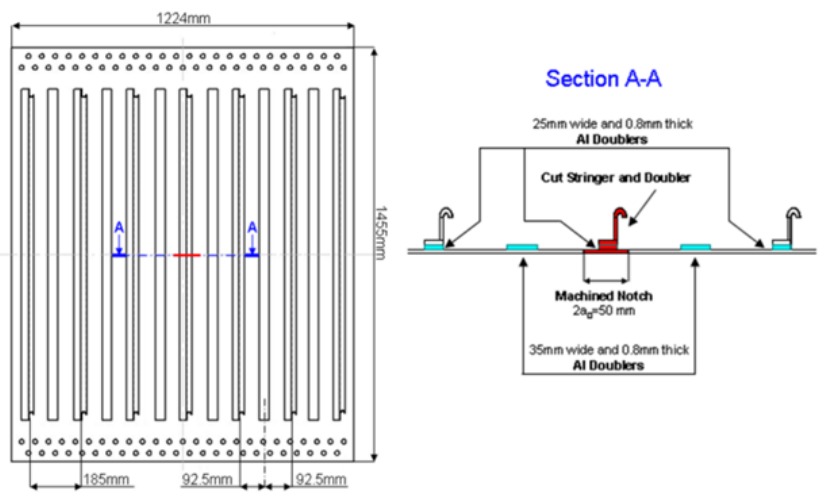

Figure 4: Representation of a "seven stringers" panel with doublers bonded between and under the stringers (a) and with an additional glass fiber reinforcement (b).

The tests were performed by means of a servo-hydraulic INSTRON 8805 machine with a 1MN load cell. The clamping was specifically designed for the 7-stringers panels (see Fig. 5). An anti-bending device was installed to prevent the out of plane deflection of the panel during the test (see Fig. 5).

The skin, like the doublers, were made of 2024-T3 aluminum alloys with 1,4 $\mathrm{mm}$ thickness the former and $0,8 \mathrm{~mm}$ the latter. The stringers were "J"-shape extruded profiles made of high strength 7349-T76511 aluminum alloy. An antibending device was installed to prevent the out-of-plane deflection of the panel during the test (see Fig 5).

In the experiments, the fatigue crack propagation was investigated starting from a through-the-thickness, $50 \mathrm{~mm}$-long, machined notch. The crack was placed across the middle stringer; this stringer and the underlying doubler (when present) were also cut. The loading parameters were the same for all the tested configurations (constant amplitude loading, $280 \mathrm{kN}$ maximum force and 0.1 load ratio). The tests ended when the crack was "four bays" long or in case of panel failure. 

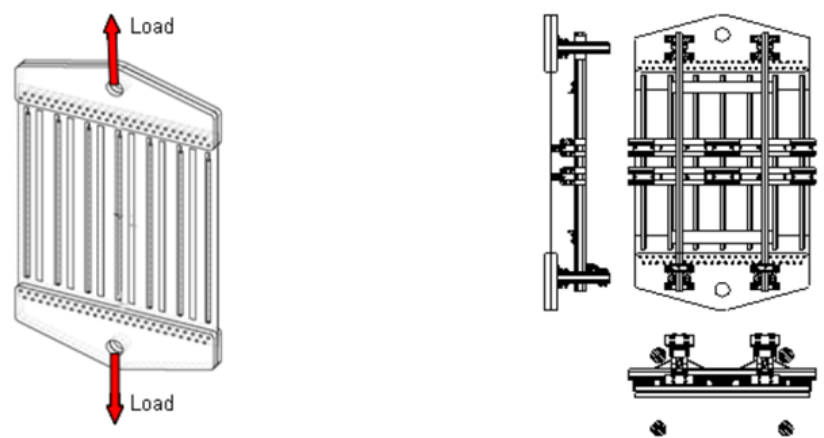

Figure 5: Representation of the clamping system (on the left) and of the anti-bending device (on the right).

The observed crack lengths within the two bays were practically symmetrical: this has permitted to draw the crack propagation curves $[a=f(N)]$ considering the average crack length between the left and right crack tip displacement (see Fig. 6).

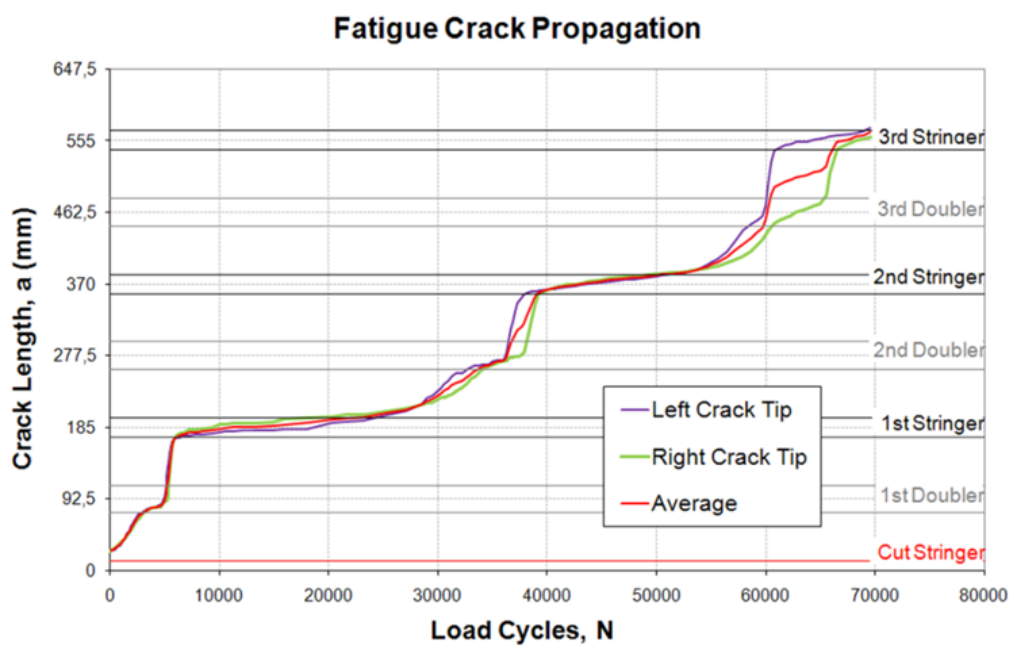

Figure 6: Experimental FCP curve illustration of left and right crack length and their average values (two bays-cracked panel).

\section{NUMERICAL MODELING}

he panels tested at EADS-IW are simulated using finite element analysis with the commercial software ABAQUS. A quarter of the panel was modeled so as to limit the computational complexity.

Two important approximations in the FE analysis are:

- the propagation occurs in the perpendicular direction to that of load application (Mode I);

- the front of the crack is assumed to be straight and modeled with two elements in the thickness (see Fig. 7).
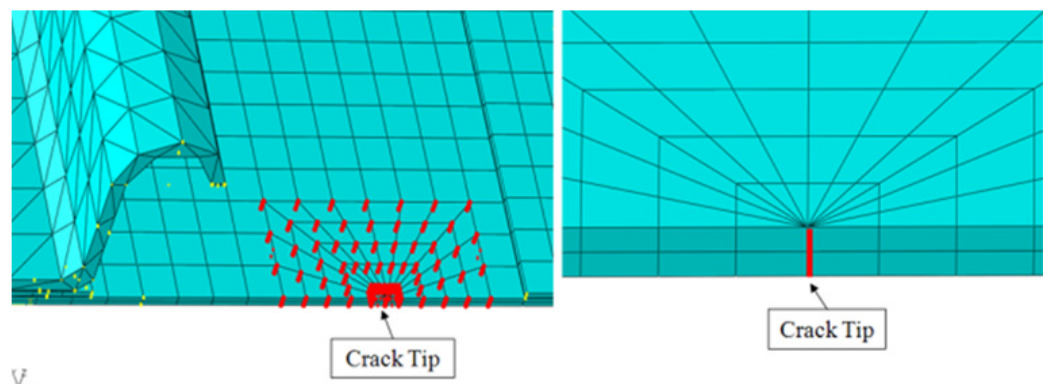

Figure 7: Straight crack front in the thickness direction. 


\section{MATERIAL PROPERTIES}

The following elastic constants were used in the FE analysis.

$\begin{array}{lll}\text { Skin and doublers: } & E=73100 \mathrm{MPa} & \text { Young's modulus } \\ & v=0,33 & \text { Poisson's ratio. } \\ \text { Stringers: } & E=71700 \mathrm{MPa} & \text { Young's modulus } \\ & v=0,33 & \text { Poisson's ratio. }\end{array}$

\section{LOADS AND CONSTRAINTS}

$\mathrm{I}$ n Fig. 8, the loads and the constraints are shown, while the bonding between the components of the panel was reproduced in Abaqus with the TIE constraint. In the simulation analysis the maximum load of the experimental tests was considered $\left(F_{\max }=280 \mathrm{kN}\right)$.

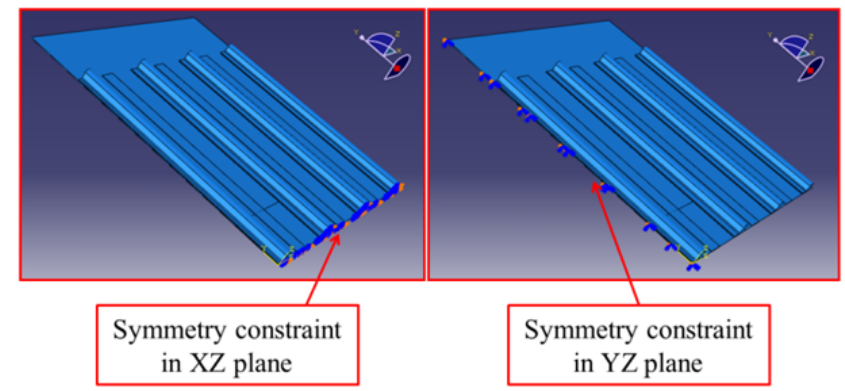

Figure 8: Loads and constraints on the quarter of the panel

\section{MODELING AND MESH}

$\mathrm{I}$ $\mathrm{n}$ the study, two typologies of element (shell and solid) and different refinements of the mesh at the crack tip were considered (see Fig. 9), namely:

- a 3D model with shell elements;

- a 3D model with solid elements (linear or quadratic geometric order)

- a 3D model with discretization by means of shell-to-solid coupling.

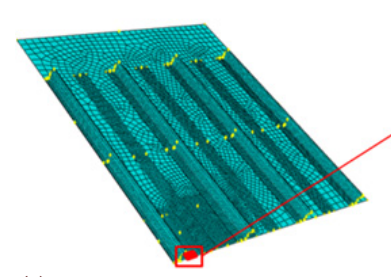

(a)
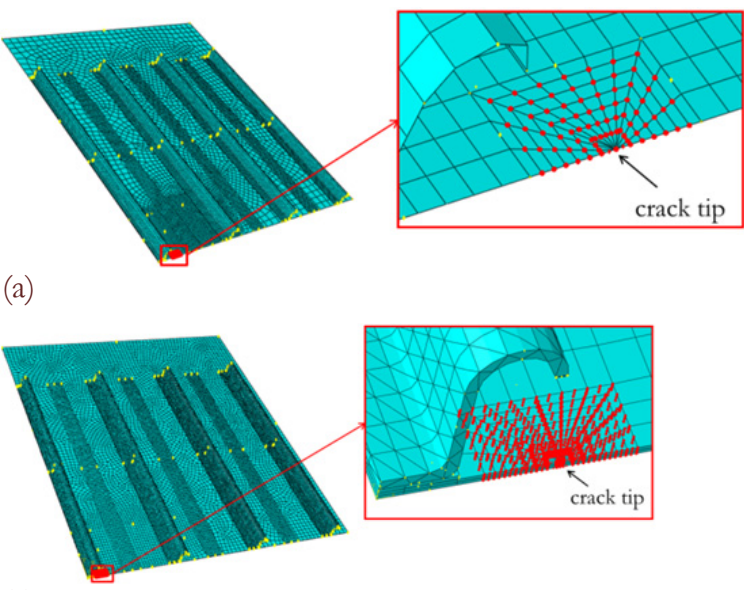

(c)

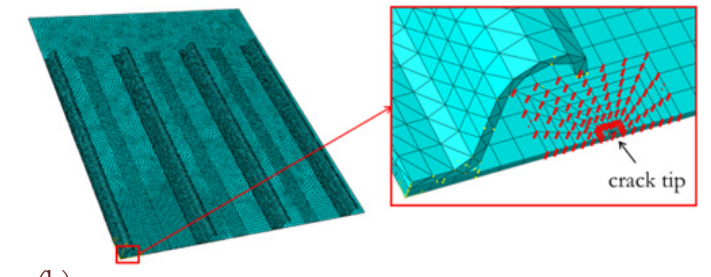

(b)

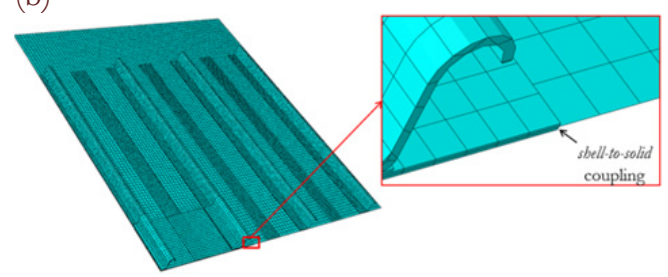

(d)

Figure 9: Different modeling typologies: second-order shell elements with collapsed Quarter-Point Node elements at the crack tip (a), first-order solid elements collapsed at the crack tip (b), second-order solid elements with collapsed Quarter-Point Node elements at the crack tip (c), same as (a) - (c) with shell-to solid coupling between solid and shell parts (d). 
Geometrically linear analyses were performed to find, for each crack length analyzed, the value of $K$ corresponding to the maximum load of the test. The stress intensity factor range $\Delta K$ was then calculated according to the load ratio used in the experiments. The Mode I, II and III stress intensity factors are calculated using the contour integral method and they showed a limited variability with the distance of the contour from the crack tip. The $K_{I I}$ and $K_{I I I}$ parameters assume always values close to zero, therefore the crack propagation is Mode I-dominated. For each size of the defect an average value of the $K$ factor was calculated across the thickness (see Fig. 10).

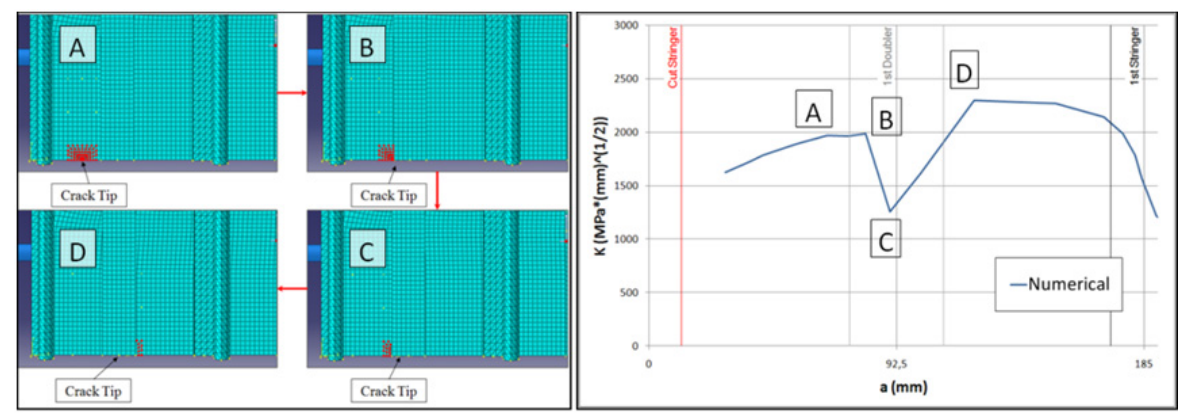

Figure 10: $\mathrm{K}$ value in 4 different positions of the crack tip across a doubler and a stiffener.

After a study of convergence based on analysis between various discretization solutions, a modeling with linear solid elements was chosen (see Tab.1), in this way through a mobile partition (see Fig. 11) with good density of elements near to the fracture tip (see Fig. 7), a good compromise between the convergence of the numerical results in terms of stress intensity factor and acceptable computation times was achieved.

\begin{tabular}{|c|c|c|c|}
\hline \multirow{2}{*}{ Element } & \multicolumn{3}{|c|}{ Characteristics } \\
\hline & $K^{1}[M P a \sqrt{ } m]$ & Process Time ${ }^{2}$ & $K$ Deviation ${ }^{3}[\%]$ \\
\hline Shell Linear & 1771.3 & 5.2 & 3.46 \\
\hline Shell Parabolic (QPNT) & 1780.5 & 33.1 & 3.99 \\
\hline Solid Linear & 1780.7 & 7.4 & 4.01 \\
\hline Solid Parabolic (QPNT) & 1712 & 100 & 0 \\
\hline \multicolumn{4}{|c|}{$\begin{array}{l}{ }^{1} \text { Values obtained by the solver taking into account the follow conditions of analysis: } \\
\text { - Same configuration of loads and constraints; } \\
\text { - Same crack length }(a=42,71 \mathrm{~mm}) \text {; } \\
\text { - Same element dimensions in the plane of extension of the skin in the panel; } \\
{ }^{2} \text { Normalized values in respect to computation time with modeling elements Solid Parabolic (QPNT) } \\
{ }^{3} \text { The deviations were assessed against the best geometric discretization (with Solid Parabolic elements - QPNT) }\end{array}$} \\
\hline
\end{tabular}

Table 1: Comparison between the discretization solutions considered in the FE study (QPNT is Quarter-Point Node Technique).

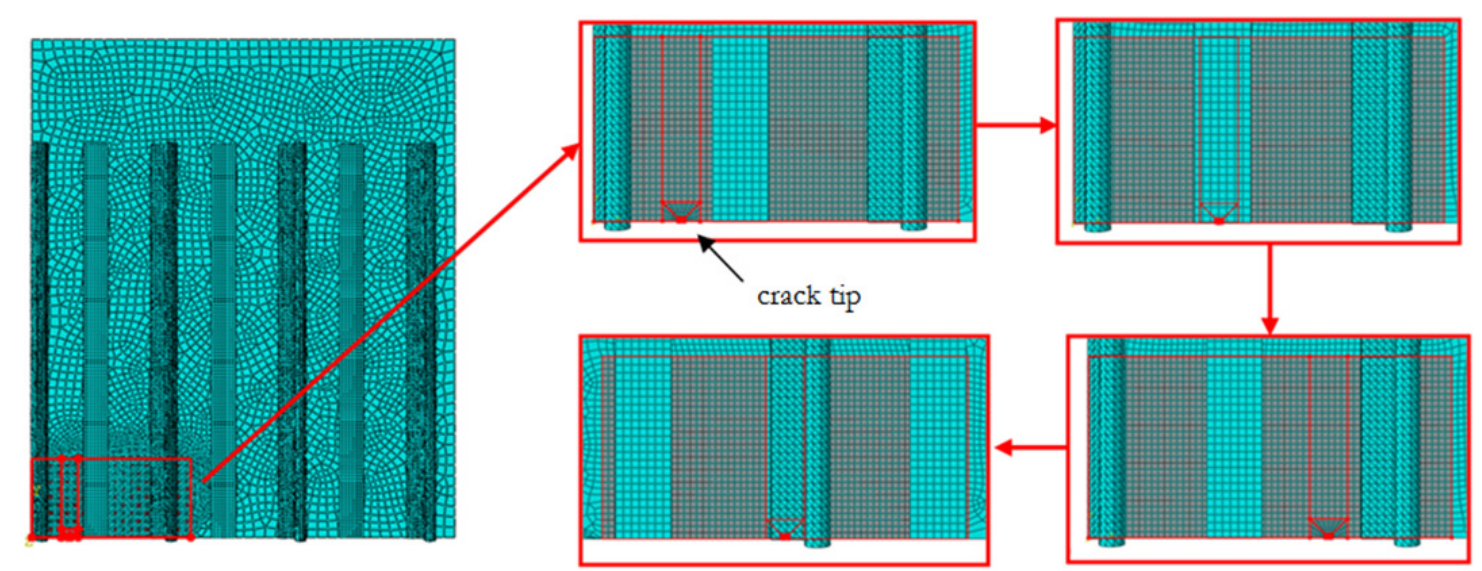

Figure 11: The mobile partition around the crack tip. 
Once chosen the modeling of the skin, stringer/doubler and crack tip, different models of skin-reinforcement coupling were considered. The main differences consist in the modeling of the presence of the adhesive or not and of the rupture of a reinforcement after the crack has passed it (see Tab.2 and Fig. 13). The presence of an anti-bending device was also reproduced (model ID 4-NB - see Fig. 12).

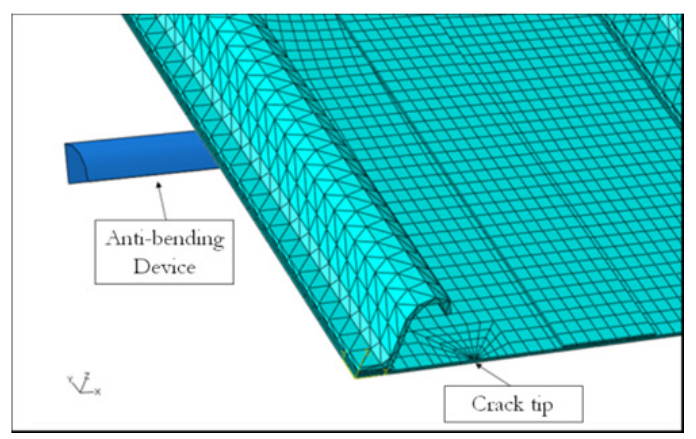

Figure 12: Representation of the anti-bending device developed in the FE modeling of panel 4-NB (No Bending).

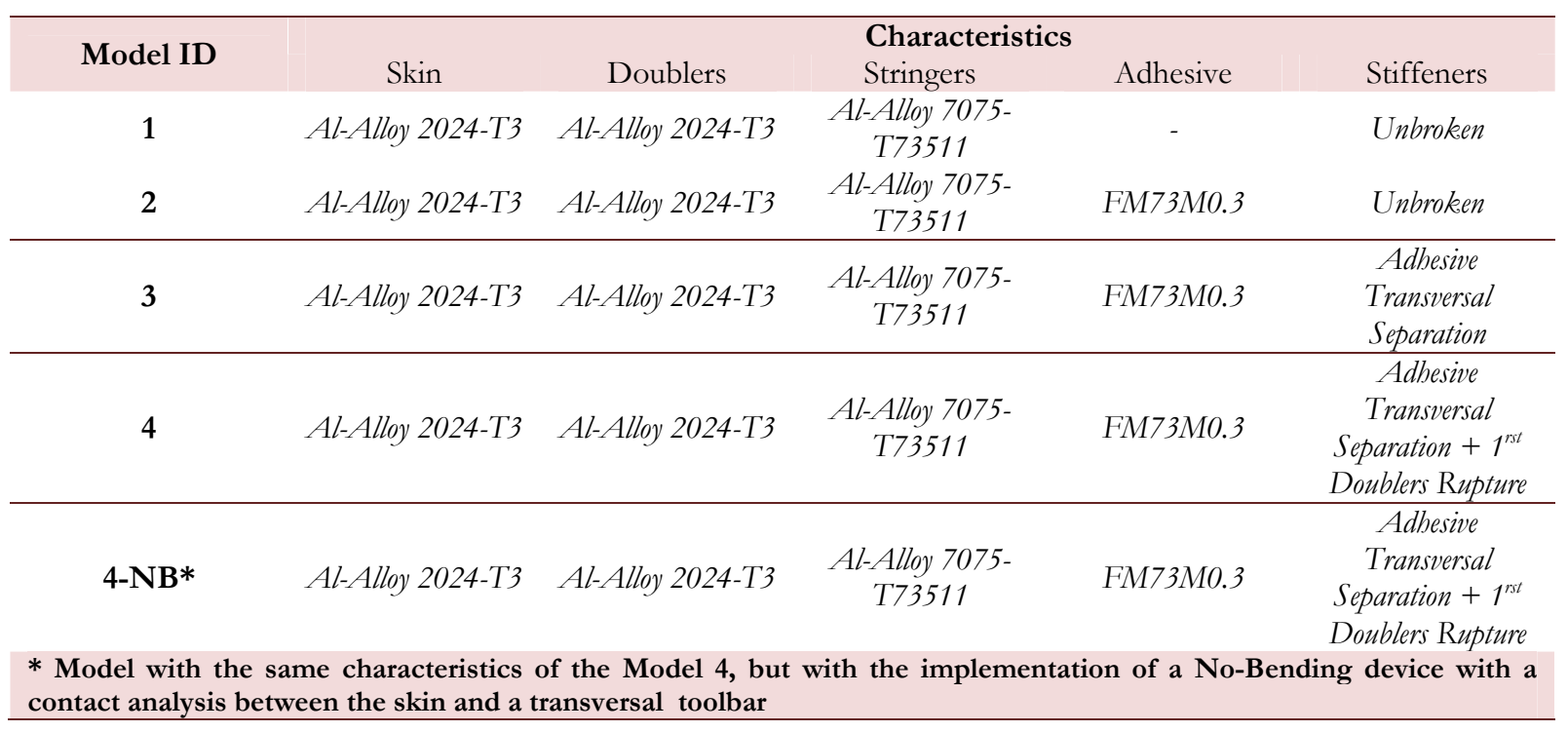

Table 2: FE models developed in this work.

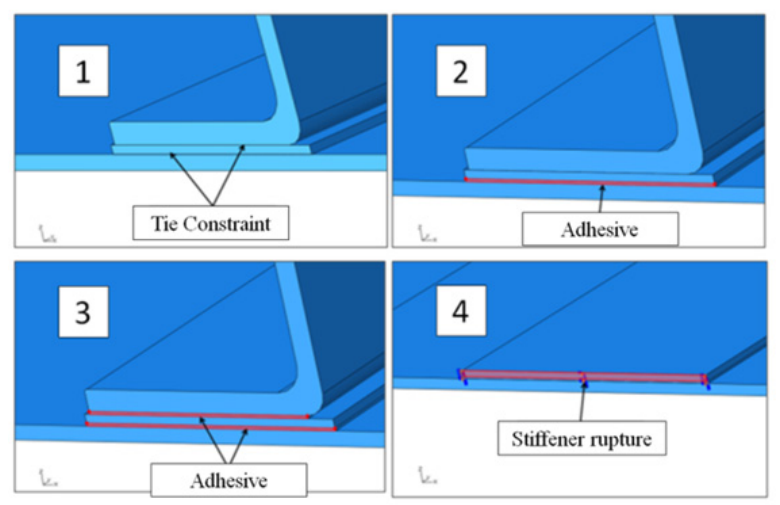

Figure 13: Representation of the different skin-stiffener couplings simulated.

To assess the number of cycles to attain a given crack length, the following procedure was considered:

- in the range of interest, the FCP data of the skin material, were approximated with a straight line in a bi-logarithmic $d a / d N-\Delta K$ plane (Paris regime); the experimental data were taken from AFGROW database [9]. 
- in the FE model, the $\mathrm{K}$ factor calculation was done in steps, then a model for each specific crack length was made (see Fig. 10). Since the crack growth rate varies significantly near the reinforcements, most of the steps were concentrated in those areas;

- the number of cycles was obtained by a numerical integration with a trapezoidal rule:

$$
N_{i, i+1}=\frac{1}{2}\left(\frac{1}{C \Delta K_{i}^{m}}+\frac{1}{C \Delta K_{i+1}^{m}}\right) a_{i, i+1}
$$

where $i$ and $i+1$ are the limits of a single increment.

Other details considered after preliminary studies are the effective load ratio at the crack tip, which changes during the crack propagation, and the effect of considering a geometric nonlinearity in the analysis of reinforced panels.

\section{RESULTS AND DISCUSSION}

$\mathrm{I}$ $\mathrm{n}$ the following figures, the analysis results are shown for each of the different models. With two elements in the skin thickness, the stress contour map at the crack tip is represented on Fig. 14.

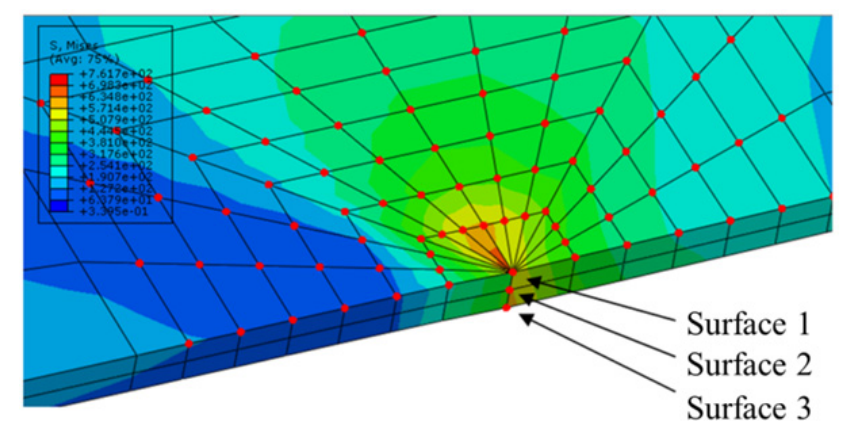

Figure 14: Node positions in seven contours chosen for the crack modeling. The number 1 is the surface where the stiffeners are bonded.

At first, the influence of the presence of the adhesive between skin and stiffeners was assessed (Model 02 vs. Model 01). Where adhesive was introduced, the final crack length is reached in a lower number of cycles (see Fig. 15). In Model 03, the separation of the adhesive under the first doubler was modeled and an increase of crack growth rate in the first bay is observed. In the panels 4 and 4-NB, the increase of $\mathrm{K}$ factors after the crack runs beyond the first stiffener is caused by the (simulated) rupture of the first doubler (see Fig. 16).

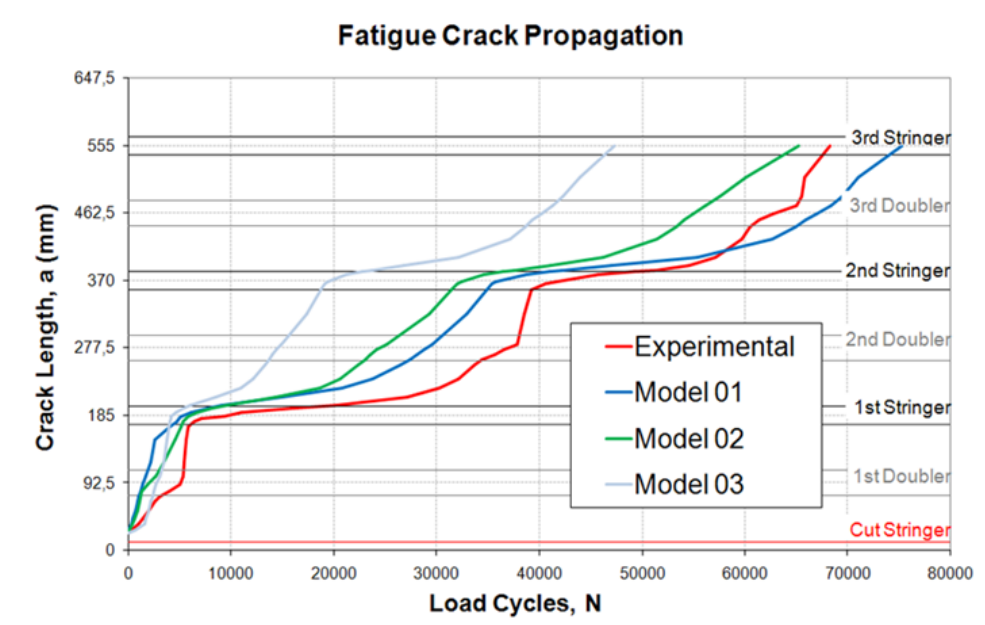

Figure 15: Comparison between experimental and numerical FCP curves (Models 01, 02 and 03). 
The Model 01 showed the most faithful reproduction of the crack propagation. The absence of the adhesive in the simulation brings in a higher rigidity near the reinforcements that apparently reproduces the stresses in the skin better than the other models. The inclusion of the anti-bending device in the model 04-NB until the second reinforcement leads at a more faithful reproduction of the initial stage of crack propagation.

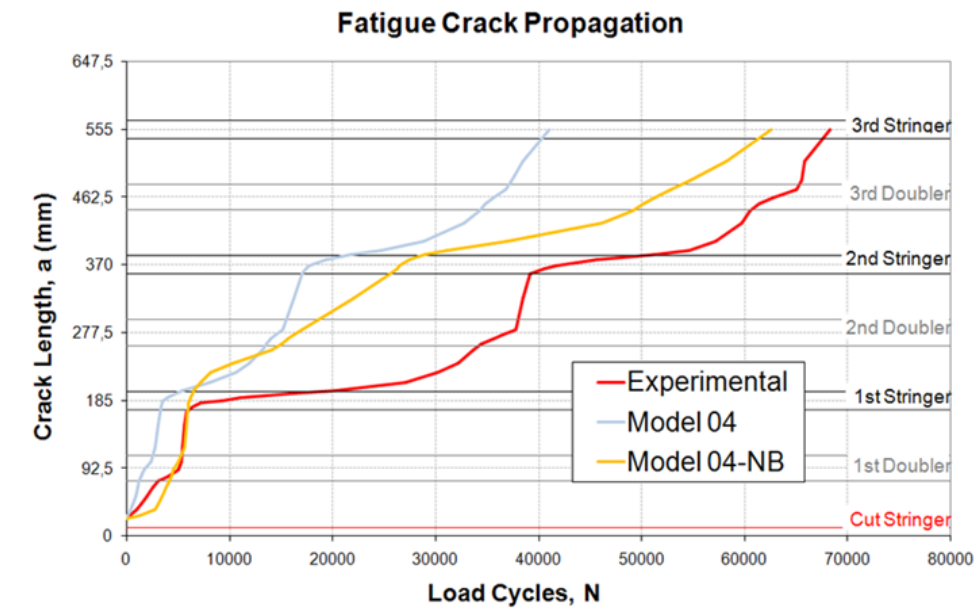

Figure 16: Comparison between experimental and numerical FCP curves (models 4 and 4-NB).

\section{CONCLUSIONS}

$\mathrm{I}$ $\mathrm{n}$ this study, the influence of several parameters that affect the crack propagation rate was evaluated and the focus was aimed at obtaining results comparable with experiments. The effect of type of skin-stiffener coupling has been simulated, with particular interest on the assessment of the presence of the adhesive and the possible debonding. The possibility of first doubler breakage was also considered. Finally, by introducing an anti-bending device, a good correspondence in the first phase of propagation was obtained, differently from the other models simulated.

\section{ACKNOWLEDGEMENTS}

he authors gratefully acknowledge Dr. Ing. Marco Pacchione, Airbus, for supplying the experimental data.

\section{REFERENCES}

[1] M. Pacchione, E. Hombergsmeier, In: Proceedings of the $1^{\text {st }}$ International Conference of Engineering Against Fracture, University of Patras, Patras, Greece (2008).

[2] Meneghin, M. Pacchione, P. Vermeer, In: $25^{\circ}$ ICAF Symposium - Rotterdam, (2009).

[3] M. B. Heinimann, R. J. Bucci, M. Kulak, M. Garratt, In: Proceedings of the 23rd ICAF Symposium, Ed. Dalle Donne C, (2005) 197.

[4] M. Heinimann, M. Kulak, R. Bucci, M. James, G. Wilson, J. Brockenbrough, H. Zonker, H. Sklyut, In: Proceedings of ICAF 24th Ed Lazzeri L, Naples, (2007) 206.

[5] M. Giglio, A.Manes, Engng Fract Mech., 75 (2008) 866.

[6] R.C. Alderliesten, International Journal of Fatigue, 31(6) (2009)1024.

[7] X Zhang, M Boscolo, D Figueroa-Gordon, G Allegri, P. E. Irving, Eng Fracture Mechanics, 76 (2009) 114.

[8] C. Mittelstedt, Composite Structures (2008).

[9] AFGROW website: http://www.afgrow.net/. 